\title{
Using Signal Processing Techniques to Enhance the Temperature Measurements Using Nanohole Array Sensors
}

\section{Masoud Modaresifar ${ }^{1}$, Gregory J Kowalski ${ }^{1 *}$ and Dale Larson ${ }^{2}$}

${ }^{1}$ Department of Mechanical and Industrial Engineering, Northeastern University, Boston, USA

${ }^{2}$ Charles Stark Draper Laboratory, Director of Biomedical Engineering, Cambridge, USA

*Corresponding author: Gregory J Kowalski, Department of Mechanical and Industrial Engineering, Northeastern University, Boston, USA, Tel: +1617 373 2971 ; Email: gkowal@coe.neu.edu

Received date: Apr 11, 2017; Accepted date: Apr 20, 2017; Published date: Apr 28, 2017

Copyright: (c) 2017 Modaresifar M, et al. This is an open-access article distributed under the terms of the Creative Commons Attribution License, which permits unrestricted use, distribution, and reproduction in any medium, provided the original author and source are credited.

\begin{abstract}
A signal processing approach to reducing noise in the observed transmission through nanohole array sensors used in a microscale calorimeter is presented. The results demonstrate that using rectification and moving average processes, a well-defined Extraordinary Optical Transmission (EOT)-temperature calibration curve can be developed in the presence of a moving light fringe pattern.
\end{abstract}

Keywords: Signal processing; Moving light fringes; Moving average; Nanohole Array Sensors (NHA); Calorimeter

\section{Introduction}

The use of Nanohole Array Sensors (NHA) to measure the temperature and concentration change in a microscale calorimeter has been described (Figure 1) [1-5].

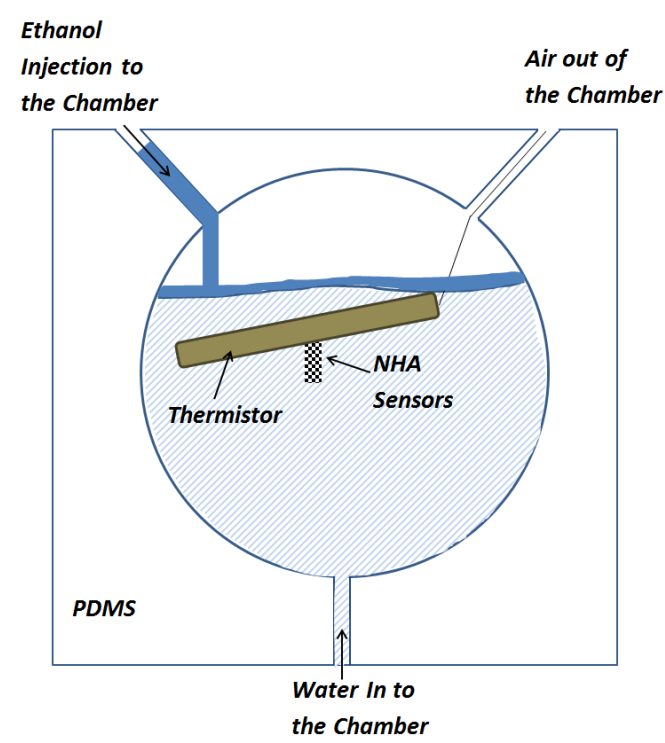

Figure 1: Schematic of the flow cell for injection experiments. The inlets, thermistor and NHA sensors locations are shown. The thermistor and NHA sensors are at the same relative position in the chamber.

Light wave fringes are observed during the reaction process (Figure 2A) involving fluids with different index of refractions [2,3]. These fringes change the incident intensity on the NHA and the observed
Extraordinary Optical Transmission (EOT). The induced EOT changes increase the signal noise (Figure $2 \mathrm{~B}$ ) and complicate obtaining an EOT-temperature calibration. Signal processing techniques are used to reduce the EOT noise introduced by these fringe patterns. The EOT noise observed in Figure 2 for the 6 sensors is series of peaks and valleys shortly after the $60 \mathrm{~s}$ injection of the ethanol into the water at $400 \mathrm{~s}$. After the injection, the response becomes monotonic because the concentration is uniform throughout the cell and the fringe patterns disappear. Each sensor has a different EOT reading because of slight differences among them (Figure 2).
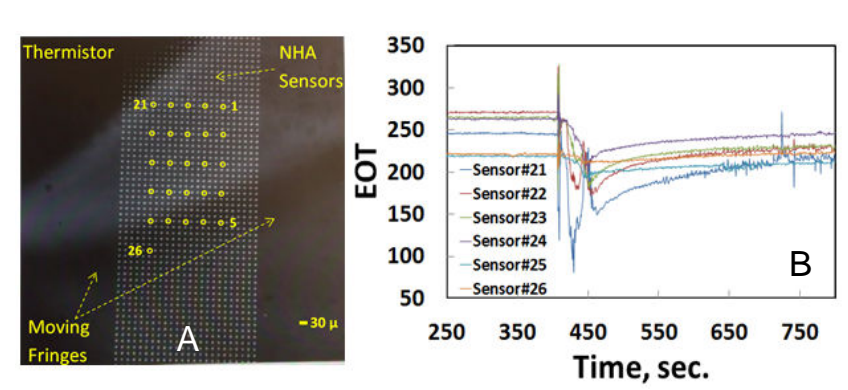

Figure 2: A) Overexposed CCD view of the NHA sensors illustrating the fringes. B) Raw EOT data before, and after a sixty s injection of ethanol into water at $400 \mathrm{~s}$.

\section{Development}

The first step in the signal processing of the EOT signal is rectifying the data. Each sensor EOT signal of pure water (time $<400 \mathrm{~s}$ ) which is relatively uniform is averaged using one hundred data points starting at $380 \mathrm{~s}$. The rectifying filter compares all the EOT data points of a sensor to its averaged EOT. The points with large EOT value relative to the average are saved. The EOT data with lower value relative to the average will be reflected above the average value with the same intensity. Figure 3 summarizes the raw data and the rectified data for a typical sensor, \#24. A moving average filter (35 data points) is also applied to the rectified data to further decrease the EOT noise and 
Citation: Masoud Modaresifar, Gregory J Kowalski* and Dale Larson (2017) Using Signal Processing Techniques to Enhance the Temperature Measurements Using Nanohole Array Sensors. Pharm Anal Acta 8: 545. doi:10.4172/2153-2435.1000545

Page 2 of 2

better prepare the signal for temperature and concentration calibration.

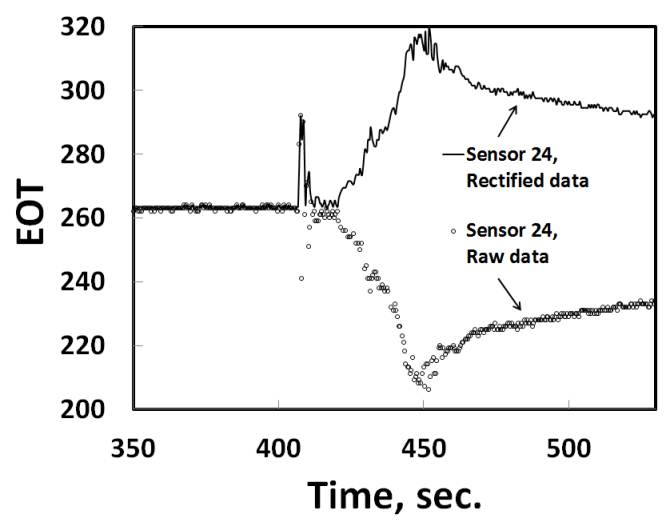

Figure 3: Raw EOT data (circle, lower line) and the rectified data (solid upper line) for sensors 24 . Rectifying the data reduces the peak to valley noise as well as yielding data that is more consistent with the thermistor measurement.

The temperature of the chamber is measured by a micro thermistor (U.S. SENSOR Corp., USP17640) that is at a similar level in the chamber (Figure 1) as the NHA and experiences similar temperature changes. The result of the final filtered EOT for the 6 sensors in Figure 2 are summarized in Figure 4. The temperature-time trend for the EOT intensity is analogous to that for the thermistor. The EOT variation as a function of the temperature variation is shown in Figure 5 for the injection period for sensor 24 . Sensor 24 is in close proximity to the thermistor. Figure 5 is used to calibrate the EOT relative to the temperature.

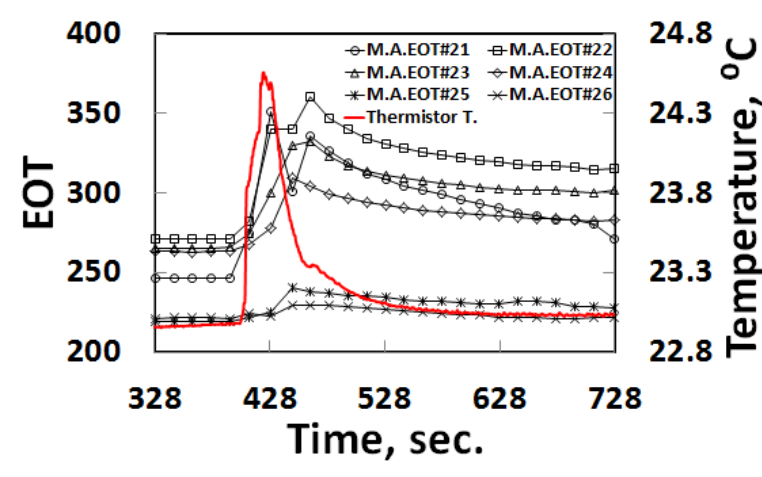

Figure 4: Comparison of the filtered EOT data for 6 selected sensors with the measured temperature from thermistor.
The dashed line represents the convolved concentration and temperature signal during the ethanol injection. The solid line is at a constant concentration and is only a function of temperature [1]. After the injection, time greater than $460 \mathrm{~s}$, the chamber cell is cooling at a constant concentration. As observed in Figure 5, the EOT variation as a function of the temperature results in a monotonic relationship that is used for calibration. The results shown in Figure 5 demonstrates the advantages of using the signal processing-rectification method with a moving average to extract more useful information from the EOT measurements than provided by the data in Figure 2 .

The consistency between the EOT and temperature measurements is better than Figure 4 that observed in Figure 2.

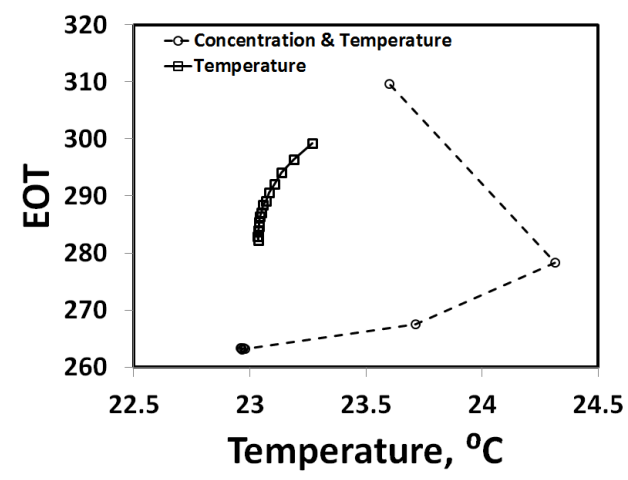

Figure 5: Temperature-EOT calibration for sensor 24. Dashed linecircle curve is for increasing temperature during dilution as the ethanol mole fraction changes from 0 to 0.103 . The region corresponding to cooling at constant concentration results in a monotonic calibration curve (line with square symbols)

\section{References}

1. Sen MA, Kowalski GJ, Fiering J, Larson D (2015) A continuous flow microfluidic calorimeter: 3-D numerical modeling with aqueous reactants. Thermochim Acta 603: 184-196.

2. Modaresifar M, Kowalski GJ (2017) Microscale Calorimetric Device for Determining Reaction Parameters. Thermochim Acta.

3. Sen M, Kowalski G, Fiering J (2011) Optical Temperature Monitoring of Microfluidic Coflow Reactions Using Nanohole Arrays.

4. Kowalski GJ, Amir T, Jin Ji J, O’Connel G, Sen M, et al. (2009) Fast Temperature Sensing Using Changes in Extraordinary Transmission Through an Array of Subwavelength Apertures. Optical Eng 48: 104402.

5. Sen M, Kowalski GJ, Fiering J, Larson D (2012) Temperature sensitivity of nanohole array sensors, ASME. International Conference on Micro/ Nanoscale Heat Transfer, ASME 2012 Third International Conference on Micro/Nanoscale Heat and Mass Transfer pp: 117-123. 